Revista Mexicana de Economía y Finanzas Nueva Época, Vol. 13 No. 4, (2018), pp. 585-601

DOI: http://dx.doi.org/10.21919/remef.v13i4.340

\title{
Impacto de la estructura tributaria sobre el crecimiento económico: el caso de México \\ Humberto Banda-Ortiz ${ }^{1}$ \\ Universidad Autónoma de Querétaro \\ Edgar Demetrio Tovar-García ${ }^{2}$
}

Universidad Panamericana

(Primera recepción: 27/abril/2017, última recepción: 28/feb/2018, aceptado: $31 /$ julio/2018)

\section{Resumen}

En este artículo se estudia empíricamente el impacto de la estructura tributaria sobre el crecimiento económico de México de 2005 a 2016. Se desarrolló un modelo econométrico usando como variables independientes: impuesto sobre la renta, impuesto al valor agregado, impuesto especial sobre la producción y servicios e impuesto sobre las importaciones. Los resultados mostraron un efecto negativo y significativo sobre el PIB per cápita del impuesto sobre la renta. Por el contrario, el impuesto al valor agregado mostró impactos positivos. Por lo tanto, la recomendación es apoyar una estructura tributaria donde el impuesto sobre la renta tenga poca relevancia. Por la falta de datos para algunas variables para años previos al 2005, el análisis está limitado al periodo de estudio enunciado. No obstante, esta es la primera investigación sobre el nexo crecimiento económico y estructura fiscal para México, aunque dicho nexo se ha estudiado en varios países desarrollados y en unos pocos países en vías de desarrollo. En línea con esa literatura, en México también se encuentra que la estructura tributaria afecta el crecimiento económico en el largo plazo.

Clasificación JEL: E62, H20, O40

Palabras clave: Estructura tributaria, crecimiento económico, impuesto sobre la renta, impuesto al valor agregado

\section{The impact of tax structure on economic growth: The case of Mexico}

\section{Abstract}

The purpose of this article is to empirically study the impact of the tax structure on the economic growth of Mexico from 2005 to 2016. An econometric model was developed using income tax, value added tax, excise duty on production and services, and import tax as independent variables. The results showed a negative and significant effect on the per capita GDP of the income tax. Conversely, the value added tax showed positive impacts. Therefore, the recommendation is to support a tax structure where the income tax has little relevance. Due to a lack of data for some variables for the years prior to 2005 , the analysis is limited to the abovementioned period of study. Nevertheless, this is the first research on the economic growth nexus and tax structure for Mexico, although said nexus has been studied in various developed countries and in a couple developing countries. In line with this literature, it is also found that in Mexico the tax structure affects economic growth in the long term.

\footnotetext{
${ }^{1}$ Profesor Investigador. Universidad Autónoma de Querétaro, C. U. Cerro de las Campanas s/n, Querétaro, Querétaro, 76010. Autor para correspondencia: humberto.banda@gmail.com

${ }^{2}$ Profesor Investigador. Universidad Panamericana. Escuela de Ciencias Económicas y Empresariales. Prolongación Calzada Circunvalación Poniente 49, Zapopan, Jalisco, 45010, México.dtovar@up.edu.mx
} 
586 REMEF (The Mexican Journal of Economics and Finance)

Impacto de la estructura tributaria sobre el crecimiento económico: El caso de México

\section{Abstract}

JEL Classification: E62, H20, O40

Keywords: Tax structure, economic growth, income tax, value-added tax

\section{Introducción}

Con la apertura comercial en México se realizaron modificaciones a las leyes fiscales con el objetivo de aumentar la competitividad internacional y ser un país atractivo para la inversión extranjera directa. Además, con el inicio de la alternancia política en el año 2000 se implementaron diferentes reformas con la finalidad de impulsar el crecimiento económico y reducir los niveles de pobreza de la población. Entre dichas reformas se encuentran la laboral, la energética, la financiera y la fiscal.

Por lo que respecta a la reforma fiscal los empresarios mexicanos, y la población en general, se plantean si las variaciones en las tasas fiscales de los impuestos a los ingresos, al capital, al trabajo y al consumo han impactado positivamente en el aumento de la actividad económica, lo que finalmente repercute en el crecimiento de México, o si por el contrario la estructura fiscal ha tenido impactos negativos. Así mismo, se cuestiona si la estructura fiscal en México ha sido, efectivamente, expansiva o restrictiva.

Como se señala más adelante en la revisión de la literatura, existen estudios previos que plantean un amplio rango de factores mediante los cuales la estructura fiscal afecta el crecimiento económico en los países de una región geográfica especifica o de un conglomerado de países que poseen ciertas características en común. No obstante, no se encontraron estudios específicos que analicen la forma en como la estructura fiscal ha impactado el crecimiento económico de México.

Consecuentemente, la presente investigación tiene como finalidad realizar un estudio empírico sobre la relación que guarda la estructura tributaria con el crecimiento económico de México en el periodo comprendido del año 2005 a la segunda mitad del año 2016. Como variables dependientes claves se utilizan el Producto Interno Bruto (PIB) per cápita a precios de 2005 y el cociente del PIB con respecto a la Población Económicamente Activa (PEA), así como sus tasas de variación trimestral.

El artículo se organiza de la siguiente manera: en la sección 2 se presenta la revisión de la literatura acerca del impacto que tiene la estructura tributaria sobre el crecimiento económico. En la sección 3 brevemente se describe la estructura fiscal en México con el fin de familiarizar al lector con la estructura tributaria del país. En la sección 4 se efectúa un análisis descriptivo de los datos utilizados, se específica el modelo econométrico y se discuten los resultados. Finalmente en la sección 5, se plantean las conclusiones.

\section{Revisión de la literatura}

El modelo neoclásico básico de crecimiento económico, cuyo principal propulsor fue Solow (1956), establece que el crecimiento económico a largo plazo depende de variables exógenas, principalmente del desarrollo tecnológico. Romer (1986) propone un modelo de crecimiento económico endógeno a largo plazo, en el cual el conocimiento es un insumo de la producción que influye en la productividad marginal de los factores productivos. Cabe mencionar que los modelos de crecimiento endógeno parten de la hipótesis de que los diferentes impuestos pueden influir en el crecimiento económico de un país o región únicamente si se presentan características determinadas en la captación tributaria y en la aplicación del gasto gubernamental. Por lo que respecta a este último se considera que 
existen dos tipos: los productivos y los improductivos. En lo referente a la captación tributaria esta puede ser no distorsionadora o distorsionadora.

El modelo planteado por Romer (1986) es fundamentalmente un modelo de crecimiento con un factor endógeno: el cambio en la tecnología. En este mismo sentido Lucas (1988) plantea tres modelos que consideran las perspectivas para la elaboración de una teoría neoclásica del crecimiento económico y el comercio internacional, haciendo énfasis en factores endógenos como la acumulación del capital físico, el cambio tecnológico, el aumento de la escolaridad y el capital intelectual. Barro (1990) hace una extensión de los modelos de crecimiento endógeno e incorpora los servicios públicos, financiados mediante los impuestos, los cuales afectan los niveles de producción y, por ende, las utilidades.

En términos generales, puede decirse que en los modelos neoclásicos ni la política fiscal ni el tamaño del estado son factores relevantes que expliquen las tasas de crecimiento de la economía en el largo plazo. No obstante, Chamley (1981) subraya el impacto que tienen los impuestos sobre los ingresos y el bienestar de los hogares en el marco del modelo de equilibrio general. Sus resultados señalan que el impacto de los impuestos sobre el bienestar de las familias dependerá de la elasticidad que exista entre los niveles de sustitución entre el capital y el trabajo en la función de producción.

Wang y Yip (1992) demostraron que en el periodo comprendido entre 1954 y 1986 la estructura tributaria fue más relevante que la tasa impositiva para explicar el crecimiento económico en Taiwán. En su modelo se muestra un impacto significativo y negativo de determinados impuestos sobre el crecimiento económico, a pesar de que el efecto total de la tributación resultó no significativo.

Lo anterior es difícil de controlar en las pruebas empíricas porque el impacto de los impuestos dependerá de cómo se gasten. Jones, Manuelli y Rossi (1993) señalan el riesgo que hay si se utiliza el gasto público como variable endógena, aunque el bienestar de las familias depende de las diferentes políticas fiscales adoptadas por el gobierno. Por el contrario, Mendoza, Milesi-Ferretti y Asea (1997) apuntan que en la práctica la política fiscal es una herramienta ineficaz para el crecimiento económico. En este mismo sentido Karras (1999), realizo un estudio sobre once países de la OCDE y encontró que no existe una relación permanente entre los diferentes impuestos y la tasa del crecimiento, lo que favorece la hipótesis planteada en los modelos neoclásicos frente a los de crecimiento endógeno.

Kneller, Bleaney y Gemmell (1999) hacen una distinción entre impuestos distorsionantes, por ejemplo el impuesto al trabajo, y no distorsionantes, por ejemplo impuestos al consumo, en 22 países miembros de la OCDE y encontraron que los impuestos distorsionantes afectan el crecimiento económico. Doménech y García (2001) señalan que los resultados empíricos soportan el argumento de que el financiamiento de un determinado nivel del gasto público debe provenir de tasas fiscales sobre el trabajo y el capital que no sean elevadas, debido a las repercusiones negativas que tendrían sobre la productividad. Cabe señalar que, en lo general, los trabajos de Jones, Manuelli y Rossi (1993), Mendoza, Milesi-Ferretti y Asea (1997) y Doménech y García (2001) coinciden en la premisa de que para que el estado financie un determinado nivel de gato público es mejor establecer un impuesto al consumo y no un impuesto sobre el trabajo o sobre el capital, debido a las repercusiones que estos dos últimos impuestos tienen sobre el mercado de trabajo y sobre la inversión productiva.

Widmalm (2001) hace un análisis del crecimiento económico para el periodo 19651990 en 23 países de la OCDE y encontró que los impuestos sobre los ingresos están negativamente correlacionados con el crecimiento, mientras que los impuestos corporativos no mostraron ninguna correlación. Además, la progresividad de los impuestos mostró un 
588 REMEF (The Mexican Journal of Economics and Finance)

Impacto de la estructura tributaria sobre el crecimiento económico: El caso de México

efecto negativo para el crecimiento económico. En este mismo sentido Gemmell (2001), apoyándose en los modelos de crecimiento endógeno establece que la política fiscal puede tener influencia en el largo plazo.

Con una muestra de 25 países industrializados, Padovano y Galli (2002) señalan que las tasas medias de impuestos provocan sesgos que pueden llevar a la conclusión de que los impuestos no tienen efectos sobre el crecimiento económico, lo anterior es debido a una posible correlación alta con el gasto público. Además, encontraron un efecto negativo sobre el crecimiento económico de los impuestos marginales efectivos y los impuestos progresivos. De manera similar, Romero de Ávila y Strauch (2003) utilizaron panel de datos para 15 países de la Unión Europea, con el objetivo de analizar el impacto de la política fiscal sobre el crecimiento económico utilizando modelos de crecimiento endógeno, y corrigiendo la posible endogeneidad en las variables fiscales utilizadas.

Por su parte, Tomljanocich (2004) examina el efecto de varios impuestos sobre el crecimiento económico y concluye que las tasas de impuestos sobre las ventas, las tasas del impuesto sobre la renta corporativa y las tasas del impuesto a la propiedad tienen poco, o nulo, impacto en la tasa de crecimiento económico. Para este autor no existe evidencia concluyente de que a mayor carga fiscal se reduzca significativamente el crecimiento económico a largo plazo. No obstante de que existe consenso de que las reducciones en la inversión e innovación, provocadas por excesivas cargas fiscales, reducen el crecimiento económico.

Lee y Gordon (2005) hallaron que las tasas de impuestos tienden a estar distorsionadas por la evasión fiscal que enfrentan muchos países y encuentran que existe una correlación negativa entre el crecimiento económico y el impuesto corporativo. En este mismo sentido Scully (2006) establece que existe una relación directa e inversa entre las cargas fiscales y las tasas de crecimiento económico, es decir, la mayor carga fiscal puede disminuir, o incrementar, la tasa de crecimiento económico. Por lo que el crecimiento económico puede ser mayor con las tasas de impuestos óptimas y, por ende, los ingresos fiscales futuros serían mayores con tasas de impuestos bajas. Myles (2009) plantea que el incremento de la tasa impositiva puede afectar de forma relevante las decisiones que tomen los diferentes agentes económicos respecto a la inversión y al consumo, pero este mismo incremento en la tasa fiscal podría no impactar significativamente al crecimiento económico.

Arnold (2008) hace un estudio con 21 países miembros de la OCDE comparando los impuestos progresivos y otros indicadores fiscales, como el impuesto sobre el consumo y el impuesto a la propiedad. En sus conclusiones se encuentra que el impuesto a los ingresos se asocia con un bajo crecimiento económico, mientras que los impuestos al consumo y a la propiedad tienden a motivar el crecimiento. En concordancia con lo anterior, Arnold et al. (2011) señalan que el crecimiento económico puede alcanzarse cambiando la estructura impositiva hacia los impuestos sobre el consumo y la propiedad inmobiliaria. Además, la reducción de impuestos sobre el ingreso estimularía la demanda, incentivaría a los trabajadores y reduciría la desigualdad en el ingreso.

Duncan y Peter (2012) muestran que los impuestos progresivos a las personas reducen la desigualdad en los ingresos, especialmente en entornos donde haya una redistribución de los ingresos. Adicionalmente, Adkisson y Mohammed (2014) realizaron un análisis en 50 estados de los Estados Unidos en el periodo 2004-2010 sobre la relación que existe entre la estructura tributaria y el crecimiento a corto plazo en el PIB per cápita real y hallaron que los efectos marginales de los diferentes impuestos no fueron similares.

En lo correspondiente a los impuestos a las ventas Li y Lin (2015) realizaron un estudio sobre el efecto que tuvieron este tipo de impuestos sobre el crecimiento económico en Estados Unidos durante los años 1960 - 2013 y encontraron un impacto negativo en el 
largo plazo, no obstante que el impacto fue positivo en el corto plazo. Por lo que respecta al impacto de los impuestos sobre bienes y servicios Hakim, Karia y Bujang (2016) hicieron un análisis en países desarrollados y en vías de desarrollo y concluyeron que mientras dichos impuestos tienen una correlación negativa con el crecimiento económico en los países en vías de desarrollo, en los países desarrollados la correlación es positiva y significativa.

Por lo que respecta a México no existen estudios recientes y específicos sobre el efecto que tiene la estructura tributaria sobre el crecimiento económico. No obstante, cabe mencionar que Mann (1980) hace una aproximación histórica al tema, describiendo como ha cambiado la estructura tributaria del país en distintas etapas de su desarrollo en el periodo 1895-1975. Posteriormente se han realizado estudios que incluyen al país como parte de una región geográfica o de un grupo de países por sus características económicas. Pero no se encontraron estudios que analicen específicamente el impacto que tiene la estructura tributaria sobre el nivel o crecimiento económico de México.

\section{La estructura tributaria en México}

En México la Constitución Política, que ocupa la máxima jerarquía en el sistema legal del país, establece en el artículo 73 fracción VII que es competencia exclusiva del H. Congreso de la Unión establecer las contribuciones necesarias tendiente a cubrir el presupuesto del Estado. La misma Constitución establece en el artículo 31 fracción IV que una de las responsabilidades de los mexicanos es coadyuvar al gasto público de la Federación, los Estados y los Municipios en donde residan de manera proporcional y equitativa de acuerdo con los criterios que marquen las leyes. Es importante señalar que, con base en el principio de proporcionalidad que señala el artículo 31fracción IV, las personas físicas y morales en México pagan sus impuestos de acuerdo con el nivel de ingreso que posean.

Cada año el Presidente de México envía al H. Congreso de la Unión la iniciativa de la ley de ingresos, la cual, una vez aprobada por el Cámara de Diputados es enviada a la Cámara de Senadores para su final aprobación y publicación. La Ley de Ingresos de la Federación tiene una vigencia de un año calendario natural. De acuerdo con la Ley de ingresos el Gobierno mexicano clasifica sus ingresos en: impuestos, aportaciones de seguridad social, contribuciones de mejoras, derechos, contribuciones de ejercicios anteriores pendientes de liquidación o pago, la deuda pública, los productos y otros.

En México los ingresos también se suelen clasificar en petroleros y no petroleros, debido a la importancia que tiene el petróleo en los ingresos del Gobierno. Los ingresos petroleros, como su nombre lo indica, son los que provienen del cobro de los impuestos, derechos y aprovechamientos del petróleo. La Figura 1 muestra la tendencia y montos de los ingresos y gastos totales, así como los ingresos petroleros y no petroleros de México trimestralmente de 2005 a 2016. Es visible que los ingresos petroleros han perdido participación en los ingresos presupuestarios desde 2008, resultado de la crisis financiera global y de la caída en los precios del petróleo. 
590 REMEF (The Mexican Journal of Economics and Finance)

Impacto de la estructura tributaria sobre el crecimiento económico: El caso de México

Figura 1. Evolución de los ingresos y gasto (2005-2016)

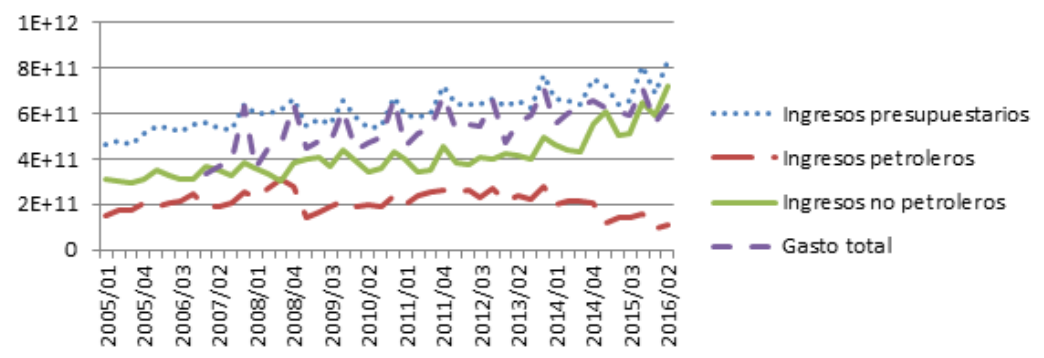

Fuente: elaboración propia con información de INEGI

Dentro de los ingresos no petroleros se incluyen los tributarios y los no tributarios (véase Figura 2). Los ingresos tributarios son aquellos que se recaudan de acuerdo con lo establecido en las leyes fiscales y los no tributarios son los que provienen del cobro de los productos, derechos, aprovechamientos y contribuciones por mejora. Las distintas clasificaciones de los ingresos gubernamentales pone de manifiesto la relevancia que tienen los impuestos provenientes de las diferentes fuentes tributarias.

Figura 2. Los ingresos en México de acuerdo con la Ley de Ingresos de la Federación

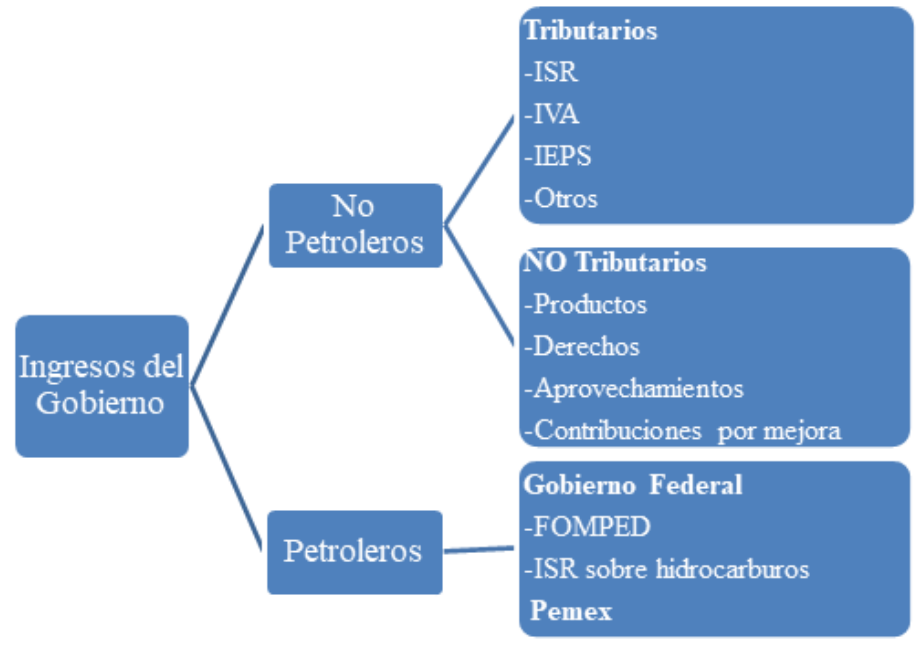

Fuente: elaboración propia

Como se puede apreciar en la Figura 2 entre los principales ingresos tributarios se encuentran el Impuesto Sobre la Renta (ISR), el Impuesto al Valor Agregado (IVA) y el Impuesto Especial sobre la Producción y Servicios (IEPS). El ISR es un impuesto directo que recae sobre las personas físicas y morales residentes en el país de acuerdo con su nivel de ingreso y actividad y grava el ingreso y las ganancias de capital. El IVA es un impuesto indirecto que grava el consumo en cada una de las etapas productivas, la comercialización y venta al consumidor final. El IEPS es otro impuesto indirecto que se aplica a la enajenación o importación de bienes que al ser consumidos por la población puedan conllevar a un gasto futuro para el Gobierno, como es el caso de las bebidas alcohólicas, cigarros y bebidas azucaradas, entre otros. 


\section{Datos, prueba empírica y resultados}

Para analizar el impacto de la estructura tributaria sobre el crecimiento económico de México se utilizaron datos trimestrales del 2005/01 al 2016/02 obtenidos de dos fuentes oficiales: Instituto Nacional de Estadística y Geografía (INEGI) y Secretaría de Hacienda y Crédito Público (SHCP). La información sobre la estructura tributaria proviene de la SHCP y los indicadores macroeconómicos sobre el PIB fueron tomados del INEGI. El periodo de estudio es resultado de una primera exploración de la información disponible por trimestre y dada la ausencia de datos para algunas variables explicativas y de control para periodos previos al 2005 se decidió acortar el periodo de estudio. Asimismo, se estimaron los valores de las variables utilizadas en pesos mexicanos y a precios reales con base en el año $2005 .^{3}$

\subsection{Variables dependientes}

Para verificar el impacto de la estructura tributaria sobre el crecimiento económico de México se utilizan cuatro variables dependientes, dos de ellas en niveles y las otras dos son sus correspondientes tasas de crecimiento. Tal como la teoría neoclásica del crecimiento económico recomienda, la variable dependiente clave es el PIB per cápita. Durante el periodo de estudio, el PIB per cápita de los mexicanos pasó de 83,918 pesos, a principios de 2005, a 102,119 pesos en el segundo trimestre del 2016. ${ }^{4}$ El promedio del periodo fue de 94,595 pesos (véase Tabla 1). La tasa de crecimiento de esta variable corresponde a su variación trimestral, que muestra mucho mayor variación que la tasa de crecimiento anualizada (véase Figura 3).

Figura 3. Tasa de crecimiento del PIB per cápita

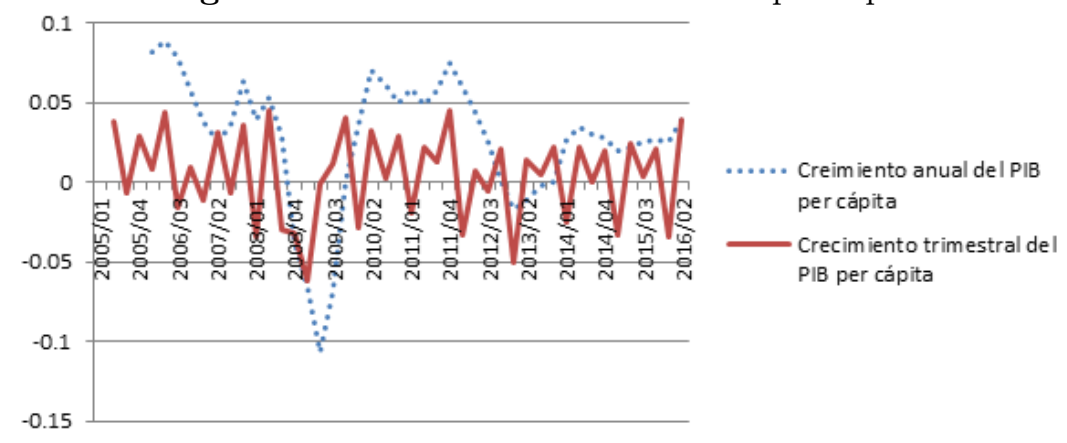

Fuente: elaboración propia con información de INEGI

La literatura empírica también recomienda evaluar el impacto de la estructura impositiva sobre la producción por trabajador, además de la producción por habitante (Arnold, 2008; Arnold et al., 2011). Por ello, se incluye como variable dependiente la razón del PIB y la población económicamente activa (PEA), lo que se puede denominar como productividad del trabajo. Durante el periodo de estudio, el PIB/PEA, es decir, la productividad de los trabajadores mexicanos pasó de 207,754 pesos, a principios de 2005, a 232,921pesos en el segundo trimestre del 2016, con un mínimo de 203,934 pesos en el tercer trimestre del 2009 y un máximo de 233,758 pesos en el segundo trimestre del 2008. El promedio del

\footnotetext{
${ }^{3}$ INEGI utiliza el año 2008 como base y en esta investigación también se analizaron los estadísticos descriptivos y se exploró los resultados del análisis de regresión utilizando las variables reales a precios de 2008. Los resultados no presentaron cambios sustanciales.

${ }^{4}$ El tipo de cambio con el dólar estadounidense para solventar obligaciones denominadas en dólares pagaderas en México al finalizar el segundo trimestre de 2016 fue de 18.46 pesos por dólar.
} 
592 REMEF (The Mexican Journal of Economics and Finance)

Impacto de la estructura tributaria sobre el crecimiento económico: El caso de México

periodo fue de 221,674 pesos (véase Tabla 1). La correspondiente tasa de crecimiento es también la variación trimestral.

Tabla 1. Estadísticos descriptivos

\begin{tabular}{lccccc}
\hline Variable & N & Media & Desviación Estándar & Mínimo & Máximo \\
\hline PIB per cápita & 46 & 94595 & 4481 & 83918 & 102119 \\
Crecimiento trimestral del & 45 & 0.005 & 0.03 & -0.06 & 0.05 \\
PIB per cápita & 46 & 221674 & 7308 & 203934 & 233758 \\
PIB/PEA (productividad) & 45 & 0.003 & 0.02 & -0.06 & 0.05 \\
Crecimiento PIB/PEA & 46 & 20690 & 1514 & 17570 & 23627 \\
$\begin{array}{l}\text { Formación bruta de capital } \\
\text { fijo per cápita }\end{array}$ & & & & \\
Crecimiento de la formación & 45 & 0.01 & 0.07 & -0.15 & 0.09 \\
bruta de capital fijo per cápita & 46 & 0.02 & 0.005 & 0.02 & 0.04 \\
IT/PIB & 46 & 0.57 & 0.06 & 0.47 & 0.68 \\
ISR/IT & 46 & 0.4 & 0.06 & 0.26 & 0.59 \\
IVA/IT & 46 & 0.003 & 0.1 & -0.3 & 0.17 \\
IEPS/IT & 46 & 0.03 & 0.01 & 0.02 & 0.04 \\
ARANCEL/IT &
\end{tabular}

Fuente: elaboración propia con información de INEGI y SHCP.

Como se puede apreciar en la Tabla 1 la relación PIB/PEA muestra más fluctuaciones que el PIB per cápita, pero la tendencia es positiva a pesar de los años de la gran crisis financiera de las hipotecas, la cual, como lo plantea Banda-Ortiz y Castañeda (2015), en el caso mexicano tiene impactos principalmente desde el tercer trimestre del 2007 y hasta el primer trimestre del 2010, no obstante que aún existen secuelas en la economía. Fácilmente se puede ver que el PIB per cápita y el PIB/PEA son indicadores similares, con una alta correlación (véase Tabla 2). En esta investigación se utilizaron ambos indicadores para desarrollar pruebas de robustez en el análisis de regresión.

Tabla 2. Coeficientes de correlación parcial (Pearson)

\begin{tabular}{|c|c|c|c|c|c|c|c|c|c|c|c|}
\hline & (1) & (2) & (3) & $(4)$ & (5) & (6) & (7) & (8) & (9) & (10) & (11) \\
\hline PIB per cápita (1) & 1 & & & & & & & & & & \\
\hline $\begin{array}{l}\text { Crecimiento trimestral del PIB } \\
\text { per cápita (2) }\end{array}$ & 0.24 & 1 & & & & & & & & & \\
\hline PIB/PEA (productividad) (3) & $0.87^{*}$ & 0.27 & 1 & & & & & & & & \\
\hline Crecimiento PIB/PEA (4) & -0.29 & -0.26 & $-0.40^{*}$ & 1 & & & & & & & \\
\hline $\begin{array}{l}\text { Formación bruta de capital } \\
\text { fijo per cápita ( } 5)\end{array}$ & $0.80^{*}$ & 0.26 & $0.73^{*}$ & $-0.46^{*}$ & 1 & & & & & & \\
\hline $\begin{array}{l}\text { Crecimiento de la formación } \\
\text { bruta de capital fijo per cápita (6) }\end{array}$ & $0.30^{*}$ & $0.85^{*}$ & $0.31^{*}$ & -0.26 & $0.48^{*}$ & 1 & & & & & \\
\hline IT/PIB (7) & $0.32^{*}$ & -0.24 & 0.19 & 0.2 & 0.01 & $-0.35^{*}$ & 1 & & & & \\
\hline ISR/IT (8) & 0.26 & -0.25 & 0.19 & -0.24 & 0.15 & -0.23 & -0.14 & 1 & & & \\
\hline IVA/IT (9) & -0.14 & 0.09 & 0.02 & -0.09 & 0.14 & 0.24 & $-0.87^{*}$ & 0.2 & 1 & & \\
\hline \multirow{2}{*}{$\begin{array}{l}\text { IEPS/IT (10) } \\
\text { ARANCEL/IT } \\
\text { (11) }\end{array}$} & -0.03 & 0.08 & -0.12 & 0.22 & -0.19 & -0.05 & $0.71^{*}$ & $-0.70^{*}$ & $-0.83^{*}$ & 1 & \\
\hline & $-0.38^{*}$ & 0.08 & -0.08 & -0.16 & 0.09 & 0.26 & $-0.66^{*}$ & -0.2 & $0.64^{*}$ & $-0.39^{*}$ & 1 \\
\hline
\end{tabular}

Fuente: elaboración propia con información de INEGI y SHCP. 


\subsection{Variables independientes}

Recapitulando brevemente lo enunciado en la revisión de la literatura, la teoría neoclásica del crecimiento económico señala que con un nivel dado de la tecnología (A), la producción $(\mathrm{Y})$, y por consiguiente el crecimiento económico, depende de la acumulación de los factores de la producción, donde el factor tierra es constante, consecuentemente depende del capital $(\mathrm{K})$ y del trabajo $(\mathrm{N})$, donde el producto marginal de los factores de la producción es positivo. Lo anterior es bien conocido en la literatura macroeconómica, y microeconómica, y se representa matemáticamente con la función de producción (1).

$$
Y=A f(K, N)
$$

El modelo también suele representarse en términos per cápita, dividiendo por el factor trabajo $(\mathrm{N})$ que en las pruebas empíricas suele aproximarse con el tamaño de la población. Como resultado, el ingreso per cápita (y) termina dependiendo exclusivamente del capital per cápita $(\mathrm{k})$ con un nivel dado en la tecnología, representado en la ecuación (2).

$$
y=A f(k)
$$

Así pues, el crecimiento económico, es decir, la variación o cambio en el ingreso per cápita está en función de la variación o cambio en el capital per cápita. En el caso de México esta variable suele aproximarse con la formación bruta de capital fijo (CAPITAL), que durante el periodo de estudio, en promedio y a precios constes, fue de 20,690 pesos per cápita (véase Tabla 1). En la Figura 4 se muestra la dinámica y tendencia del CAPITAL per cápita y del PIB per cápita, como se puede observar existe tendencia positiva en ambas variables, pero mucho más marcada en el caso del PIB, que también muestra una clara caída a partir del año 2008 (lo que corresponde a la última gran crisis).

Figura 4. PIB per cápita y formación bruta de capital fijo per cápita

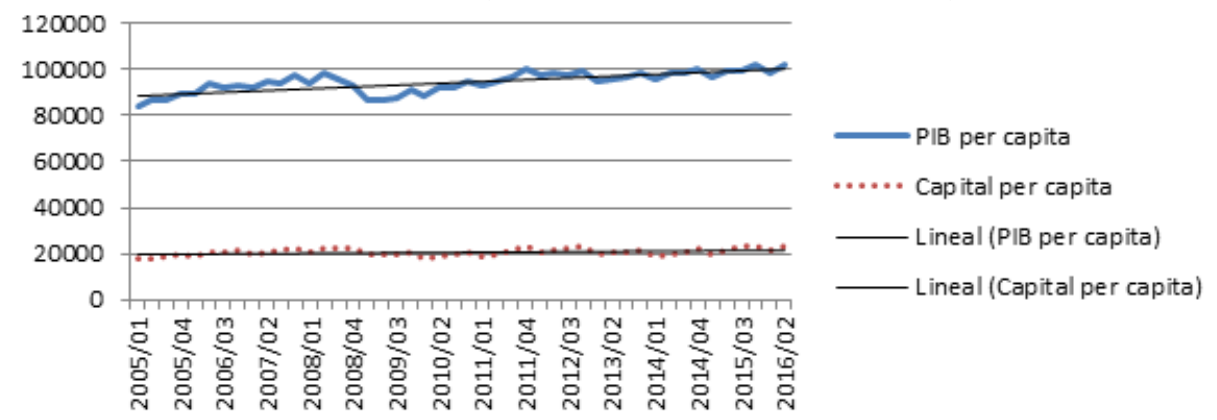

Fuente: elaboración propia con información de INEGI

A partir de este modelo básico muchas han sido las modificaciones y las extensiones del mismo para identificar otros determinantes claves del crecimiento económico, por ejemplo, el capital humano (Mankiw, Romer, y Weil, 1992), la innovación (Jones, 1995), y la apertura comercial (Edwards, 1998). En esta investigación se incluyen indicadores para aproximar estas variables, pero solo son utilizadas como variables de control y por ello no son descritas ni analizadas en profundidad.

De mayor relevancia para la presente investigación es la inclusión de la estructura tributaria en el modelo neoclásico del crecimiento económico, donde es factible utilizar distintos tipos de impuestos dependiendo del país bajo estudio. En el caso de México, son dos los impuestos que sobresalen por su contribución a los ingresos gubernamentales: el ISR y el IVA, ambos impuestos representan aproximadamente el $85 \%$ de los ingresos 
594 REMEF (The Mexican Journal of Economics and Finance)

Impacto de la estructura tributaria sobre el crecimiento económico: El caso de México

tributarios. No obstante, también son significativos el IEPS y el impuesto a las importaciones (ARANCEL). El IEPS, como ya se mencionó, grava la compra-venta de productos que generan externalidades negativas o son un costo para la sociedad, por ello el impuesto es coloquialmente conocido como un impuesto al pecado.

Estos cuatro impuestos (ISR, IVA, IEPS y ARANCEL), representan aproximadamente el $95 \%$ de los ingresos tributarios de México. Su dinámica y la contribución de cada uno de ellos a los impuestos totales ha variado durante el periodo de estudio, a excepción de los impuestos a las importaciones (véase Figura 5). Nótese que el IEPS puede ser negativo debido al efecto de la compensación. Para los fines de esta investigación se calcularon los impuestos totales (IT) como la suma de estos cuatro impuestos y posteriormente se calculó la proporción que cada uno de ellos representa con respecto a ese total (véase Tabla 1). Son estas proporciones las que permiten medir la estructura tributaria mexicana.

Figura 5. Estructura tributaria

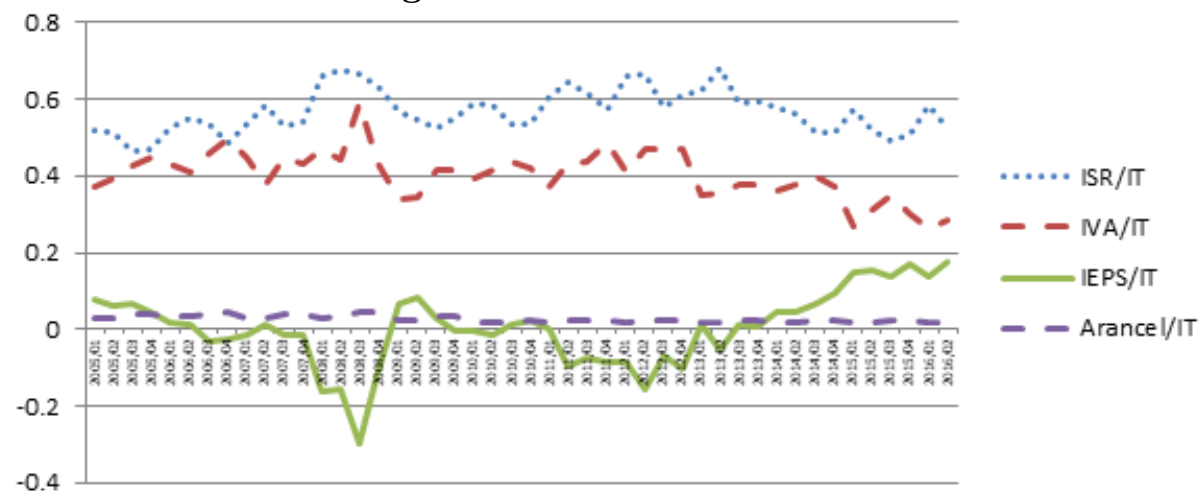

Fuente: elaboración propia con información de INEGI

\subsection{Especificación econométrica}

Dado el modelo neoclásico de crecimiento económico y las especificaciones econométricas previas que incluyen la estructura tributaria como variable explicativa, para el caso de México, el modelo básico a estimar está representado por la ecuación (3):

$$
\begin{gathered}
\text { LnPIB_per_capita } a_{t}=\beta_{0}+\Theta_{1} L n C A P I T A L \_p e r \_ \text {capita }_{t-1}+{ }_{1} \\
I S R / I T_{t-1}\left(\text { or }_{-2} I V A / I T_{t-1}\right)+{ }_{3} I E P S / I T_{t-1}+{ }_{4} A R A N C E L / I T t-1+X_{t}^{\prime} \alpha+u_{t}
\end{gathered}
$$

Las variables empleadas en la ecuación (3) fueron descritas en la sección previa, a excepción de $\mathrm{X}$, que incluye variables de control (que se enuncian posteriormente) para realizar pruebas de robustez y para corregir autocorrelación, un supuesto calve en el método de mínimos cuadrados utilizado en la estimación de los parámetros. Nótese que el PIB per cápita y CAPITAL per cápita son incluidos en términos logarítmicos, consecuentemente el coeficiente estimado proporciona la elasticidad (cambio porcentual en el PIB por unidad de cambio porcentual en el CAPITAL), esta estrategia también favorece la linealidad entre las variables. En este tipo de modelos la doble causalidad es evidente (Tosun y Abizadeh, 2005), por ello las variables independientes son introducidas con un rezago. ${ }^{5}$

\footnotetext{
${ }^{5}$ Lo deseable sería utilizar variables instrumentales. Sin embargo, la carencia de datos y variables que puedan fungir como instrumentos es una limitante para el uso de esta aproximación. En la literatura
} 
Dado que los impuestos totales (IT) son la suma de los cuatro impuestos objeto de estudio, no es posible incluir en una misma regresión los cuatro impuestos, uno de ellos debe fungir como referencia, con ello además evitamos problemas de multicolinealidad porque hay correlaciones relevantes entre estas variables (véase Tabla 2). El IVA e ISR representan los impuestos más grandes, de mayor recaudación, consecuentemente en el análisis de regresión se incluye uno u otro para evaluar el impacto de la estructura tributaria con respecto al impuesto excluido en la regresión.

La Tabla 3 presenta los principales resultados cuando las variables dependientes son el PIB per cápita y su tasa de crecimiento. Las columnas (1) y (3) muestran la especificación más sencilla, donde el CAPITAL per cápita es la principal variable explicativa (de acuerdo al modelo neoclásico) y como variable de control se introduce la razón entre ingresos totales y PIB (IT/PIB), cuyo impacto en la variable dependiente es incierto debido a que dependerá de cómo se gaste dicho ingreso, lo cual sobrepasa los límites de esta investigación, pero su inclusión como variable de control es recomendado en la literatura empírica (Arnold, 2008). En la columna (1) se evalúa el impacto de la estructura tributaria con referencia al IVA y en la columna (3) con referencia al ISR.

En lo general, los resultados coinciden con los hallazgos empíricos más recientes (Adkisson y Mohammed, 2014; Arnold, 2008; Arnold et al., 2011; Hakim et al., 2016; Li y Lin, 2015). Las estructuras tributarias donde hay más participación de los impuestos sobre el ingreso, en el caso mexicano el ISR, tienen un impacto negativo sobre el PIB per cápita. El coeficiente de ISR/IT es negativo y estadísticamente significativo al $5 \%$. Arnold (2008) y Arnold et al., (2011), con una muestra de 21 países de la OCDE que no incluye a México, encontraron que los impuestos al ingreso están asociados con una menor tasa de crecimiento económico. Este resultado también se encontró en Estados Unidos al estudiar la estructura impositiva de sus 50 estados miembros (Adkisson y Mohammed, 2014).

Por el contrario, los impuestos al consumo tienen un impacto positivo, el coeficiente estimado para el IVA/IT es positivo y estadísticamente significativo al $5 \%$. Lo que también coincide con los hallazgos previos para los países de la OCDE (Arnold, 2008; Arnold et al., 2011). Sin embargo, también hay evidencia de que los impuestos sobre los bienes y servicios están asociados negativamente con el crecimiento económico en el caso de las naciones en vías de desarrollo, así lo señalan los resultados del análisis de regresión elaborados por Hakim et al., (2016), usando una muestra de 47 países donde no se incluye a México. Por el contrario, sus resultados sugieren una asociación positiva para el caso de las naciones desarrolladas. Consecuentemente, los resultados del análisis de regresión aquí presentado sugieren que los impactos de la estructura tributaria de México se parecen a los que se observan en las naciones desarrolladas.

En el caso del IEPS, que podría considerarse como un impuesto al consumo, la evidencia es inconsistente. El IEPS/IT con respecto al IVA/IT tiene un impacto negativo y significativo sobre el PIB per cápita, pero su impacto pierde significancia estadística cuando el impuesto de referencia es el ISR. No hay estudios previos que analicen el impacto de impuestos semejantes al IEPS mexicano, por lo tanto, en la literatura no hay puntos de referencia para poder inferir y analizar los resultados aquí encontrados, que además carecen de robustez y no posibilitan una discusión profunda del impacto de este tipo de impuesto.

Por su parte, los impuestos a las importaciones (ARANCEL/IT), con respecto al IVA, tienen impactos negativos y significativos sobre el PIB per cápita, lo que concuerda con

empírica se han utilizado como variables instrumentales los rezagos de las variables independientes, pero estos instrumentos internos pueden generar problemas de especificación y por ello es factible simplemente incluir las variables independientes con rezagos. 
596 REMEF (The Mexican Journal of Economics and Finance)

Impacto de la estructura tributaria sobre el crecimiento económico: El caso de México

la ortodoxia en la teoría del comercio internacional, que predice que el libre comercio y la globalización económica promueven el crecimiento económico. ${ }^{6}$ No obstante, el impacto de los aranceles sobre el crecimiento económico de México es incierto, porque ARANCEL/IT pierde significancia estadística cuando el impuesto de referencia es el ISR. Lee y Gordon (2005), en su estudio de corte transversal con hasta 70 países, tampoco encontraron impactos estadísticamente significativos de la tasa arancelaria sobre la tasa de crecimiento del PIB per cápita. Por su parte, Hakim, et. al., (2016) encontraron que los impuestos al comercio internacional tienen un impacto positivo sobre el PIB per cápita en los países en vías de desarrollo, por el contrario, no se encuentran efectos significativos en las regresiones que incluyen a los países desarrollados.

Como era de esperarse, las regresiones básicas en las columnas (1) y (3) de la Tabla 3 presentan problemas de autocorrelación, un problema típico en las series de tiempo (véase el estadístico Durbin-Watson y la prueba Breush-Godfrey). Para verificar la robustez de los resultados enunciados anteriormente se corrigió el problema de autocorrelación introduciendo la variable dependiente rezagada como independiente. Esta es una estrategia habitual para corregir la correlación serial y además una estrategia frecuente en las pruebas empíricas previas para evaluar el impacto de la estructura tributaria (Adkisson y Mohammed, 2014; Arnold, 2008; Hakim et al., 2016; Li y Lin, 2015). Con esta modificación, los hallazgos sobre el impacto de la estructura tributaria son básicamente los mismos, véase columnas (2) y (4), el coeficiente de determinación r-cuadrada ajustada se incrementa de 0.56 a 0.76 , pero el CAPITAL per cápita cambia de signo. Lo anterior indica que el PIB per cápita trimestral es una variable que sigue una tendencia temporal y un proceso no estacionario (previsiblemente). No obstante, los residuos de las regresiones siguen un proceso estacionario, de acuerdo con las pruebas de raíz unitaria, por lo que podemos confiar en los resultados de las regresiones.

En las columnas (5) y (6) de la Tabla 3 se presentan los resultados de regresión añadiendo tres variables de control en términos logarítmicos y una variable dicotómica (dummy) que toma el valor de 1 para los trimestres comprendidos entre el tercer cuarto de 2007 y el primer cuarto de 2009 correspondientes al periodo más intenso de la crisis financiera global. La primera variable de control es la apertura comercial (importaciones más exportaciones como porcentaje del PIB), la segunda variable de control es el gasto público en ciencia y tecnología como porcentaje del PIB, y la tercera variable de control es el porcentaje de la población con educación medio superior y superior, que permite aproximar el capital humano. Los resultados de las regresiones no contradicen los hallazgos previos, es decir, el ISR impacta negativamente sobre el PIB per cápita y por el contrario el IVA lo hace positivamente.

Las regresiones de las columnas (1) a (6) corresponden a pruebas del impacto de la estructura tributaria sobre los niveles del PIB per cápita. Las pruebas empíricas sobre la tasa de crecimiento se presentan en las columnas (7) a (12), donde se replican las especificaciones econométricas previas utilizando como variable dependiente la variación trimestral del PIB per cápita y como variable de control primordial la variación trimestral de la formación de capital fijo (crecimiento del CAPITAL per cápita), sin transformación logarítmica porque pueden tomar valores negativos.

En el caso de la tasa de crecimiento del PIB per cápita los resultados no favorecen los hallazgos previos y hay muy pocas variables independientes con significancia estadística. En cuanto a la estructura tributaria solamente el IEPS/IT entra en un par de regresiones con un coeficiente positivo y estadísticamente significativo (ver columnas 9 y 10), pero

\footnotetext{
${ }^{6}$ Aunque en el caso de América Latina la evidencia empírica no favorece la teoría (Tovar, 2011; TovarGarcía, 2012).
} 
esta condición no prevalece en el resto de las especificaciones econométricas, por lo tanto, el resultado no es robusto y no es posible señalar que la estructura tributaria tiene impactos en el crecimiento trimestral del PIB per cápita. Más aún, ninguna variable incluida en las regresiones es capaz de predecir la variación trimestral del PIB per cápita. Los resultados indican que la variable(s) clave(s) para predecir la tasa de crecimiento de México aún está por encontrarse.

Tabla 3. Resultados del análisis de regresión: PIB per cápita contra estructura

\begin{tabular}{|c|c|c|c|c|c|c|c|c|c|c|c|c|}
\hline & \multicolumn{6}{|c|}{$\begin{array}{l}\text { Variable dependiente: } \\
\text { PIB per cápita }\end{array}$} & \multicolumn{6}{|c|}{$\begin{array}{l}\text { Variable dependiente: } \\
\text { Crecimiento del PIB per cápita }\end{array}$} \\
\hline & -1 & -2 & -3 & -4 & -5 & -6 & -7 & -8 & -9 & -10 & -11 & -12 \\
\hline $\begin{array}{c}\text { Dependiente } \\
\text { rezagada }\end{array}$ & & $1.04^{* * *}$ & & $1.04^{* * *}$ & $1.08 * * *$ & $1.08^{* * *}$ & & -0.3 & & -0.3 & -0.6 & $\begin{array}{ll}-0.6 \\
\end{array}$ \\
\hline \multicolumn{13}{|l|}{$\begin{array}{l}\text { Variables } \\
\text { Neoclásicas }\end{array}$} \\
\hline $\begin{array}{l}\text { CAPITAL per } \\
\text { cápita }\end{array}$ & $0.28^{* * *}$ & $-0.26^{* *}$ & $0.28^{* * *}$ & $-0.26^{* *}$ & $-0.56^{* * *}$ & $-0.56^{* * *}$ & & & & & & \\
\hline $\begin{array}{c}\text { Crecimiento del } \\
\text { CAPITAL per cápita }\end{array}$ & & & & & & & $-0.18^{* * *}$ & -0.08 & $-0.18^{* * *}$ & -0.08 & 0.12 & 0.12 \\
\hline \multicolumn{13}{|l|}{$\begin{array}{l}\text { Variables de la } \\
\text { estructura tributaria }\end{array}$} \\
\hline IT/PIB & $6.02^{* * * *}$ & $3.76^{* *}$ & $6.02^{* * *}$ & $3.76^{* *}$ & 2.35 & 2.35 & 0.33 & -0.11 & 0.33 & -0.11 & 1.38 & 1.38 \\
\hline ISR/IT & $-0.41^{* *}$ & $-0.28^{*}$ & & & $-0.33^{*}$ & & -0.23 & -0.23 & & & -0.24 & \\
\hline$\frac{15 n / 11}{\text { IVA/IT }}$ & & & $0.41^{* *}$ & $0.28^{*}$ & & $0.33^{*}$ & & & 0.23 & 0.23 & & 0.24 \\
\hline IEPS/IT & $-0.41^{\text {****}}$ & $-0.24^{* *}$ & -0.0005 & 0.04 & $-0.25^{*}$ & 0.08 & -0.09 & -0.07 & $0.15^{*}$ & $0.16^{* *}$ & -0.14 & 0.1 \\
\hline ARANCEL/IT & $\frac{-0.410 * *}{-3.07^{* * *}}$ & $\begin{array}{l}-0.24 \\
-0.38\end{array}$ & $-2.66^{* * * * *}$ & $\frac{0.04}{-0.1}$ & $\begin{array}{c}-0.25 \\
0.22\end{array}$ & 0.05 & $\begin{array}{l}-0.09 \\
-0.86\end{array}$ & $\begin{array}{l}-0.01 \\
-1.05\end{array}$ & $\frac{0.10}{-0.63}$ & $\frac{0.10}{-0.82}$ & $\begin{array}{l}-2.164^{*} \\
\end{array}$ & $\frac{1.4}{-2.4}$ \\
\hline \multicolumn{13}{|l|}{$\begin{array}{l}\text { Otras variables } \\
\text { de control }\end{array}$} \\
\hline $\begin{array}{l}\text { Apertura } \\
\text { comercial }\end{array}$ & & & & & $0.27^{*}$ & $0.27^{*}$ & & & & & -0.01 & -0.01 \\
\hline $\begin{array}{l}\text { Gasto público } \\
\text { en I-D }\end{array}$ & & & & & -0.01 & -0.01 & & & & & 0.03 & 0.03 \\
\hline Capital humano & & & & & -0.01 & -0.01 & & & & & -0.17 & -0.17 \\
\hline Crisis (dummy) & & & & & 0.03 & 0.03 & & & & & -0.004 & -0.004 \\
\hline Constante & $8.90^{* * *}$ & $2.30^{*}$ & $8.49^{* * *}$ & $2.02^{*}$ & $4.77^{* *}$ & $4.44^{* *}$ & 0.15 & $0.17^{*}$ & -0.08 & -0.06 & 0.03 & $\frac{-0.00+1}{-0.21}$ \\
\hline Se excluye & IVA/IT & IVA/IT & $\mathrm{ISR} / \mathrm{IT}$ & $\mathrm{ISR} / \mathrm{IT}$ & IVA/IT & $\mathrm{ISR} / \mathrm{IT}$ & IVA/IT & IVA/IT & $\mathrm{ISR} / \mathrm{IT}$ & $\mathrm{ISR} / \mathrm{IT}$ & IVA/IT & ISR/IT \\
\hline R-cuadrada & 0.61 & 0.79 & 0.61 & 0.79 & 0.78 & 0.78 & 0.26 & 0.28 & 0.26 & 0.28 & 0.4 & 0.4 \\
\hline $\begin{array}{c}\text { R-cuadrada } \\
\text { ajustada }\end{array}$ & 0.56 & 0.76 & 0.56 & 0.76 & 0.7 & 0.7 & 0.16 & 0.16 & 0.16 & 0.16 & 0.17 & 0.17 \\
\hline Durbin-Watson & 1.25 & 2.27 & 1.25 & 2.27 & 1.85 & 1.85 & 2.23 & 1.94 & 2.23 & 1.94 & 1.9 & 1.9 \\
\hline $\begin{array}{c}\text { Breush-Godfrey } \\
\text { (p-value) }\end{array}$ & $9.26(0.002)$ & $1.60(0.20)$ & $9.26(0.002)$ & $1.60(0.20)$ & $0.09(0.76)$ & $0.09(0.76)$ & $2.15(0.14)$ & $0.08(0.77)$ & $2.15(0.14)$ & $0.08(0.77)$ & $0.33(0.56)$ & $0.33(0.56)$ \\
\hline $\begin{array}{c}\text { Jarque-Bera } \\
\text { (p-value) }\end{array}$ & $1.02(0.60)$ & $3.23(0.19)$ & $0.62(0.73)$ & $2.81(0.24)$ & $1.69(0.42)$ & $1.69(0.42)$ & $0.19(0.90)$ & $0.43(0.80)$ & $0.19(0.90)$ & $0.11(0.94)$ & $0.32(0.85)$ & $0.32(0.85)$ \\
\hline Observaciones & 45 & 45 & 45 & 45 & 37 & 37 & 44 & 44 & 44 & 44 & 37 & 37 \\
\hline
\end{tabular}

Fuente: Elaboración propia

Dados estos resultados, en el caso de México, hay evidencia de que la estructura tributaria tiene impactos en los niveles del PIB per cápita, pero no sobre la tasa de crecimiento del mismo. Para profundizar y corroborar la robustez de estos hallazgos se realizaron los mismos ejercicios econométricos utilizando como variable dependiente la razón entre el PIB trimestral y la población económicamente activa (PIB/PEA), productividad, así como su tasa de crecimiento. La Tabla 4 muestra los principales resultados.

Se encuentra que el ISR tiene impactos negativos sobre la productividad (PIB/PEA), en las columnas (1), (2) y (5) de la Tabla 4 el coeficiente de ISR/IT es negativo y estadísticamente significativo. Por su pate los coeficientes del IVA/IT son positivos y estadísticamente significativos (ver columnas 3,4 y 6). Estos resultados concuerdan con los hallazgos previos, por lo tanto, es factible señalar que en el caso de México el ISR tiene impactos negativos en los niveles del PIB per cápita y en la productividad (PIB/PEA), por el contrario el IVA tiene impactos positivos. 
598 REMEF (The Mexican Journal of Economics and Finance)

Impacto de la estructura tributaria sobre el crecimiento económico: El caso de México

Tabla 4. Resultados del análisis de regresión: PIB/PEA (productividad) contra estructura tributaria

\begin{tabular}{|c|c|c|c|c|c|c|c|c|c|c|c|c|}
\hline & \multicolumn{6}{|c|}{$\begin{array}{l}\text { Variable dependiente: } \\
\text { PIB/PEA per cápita }\end{array}$} & \multicolumn{6}{|c|}{$\begin{array}{c}\text { Variable dependiente: } \\
\text { Crecimiento del PIB/PEA }\end{array}$} \\
\hline & -1 & -2 & -3 & -4 & -5 & -6 & -7 & -8 & -9 & -10 & -11 & -12 \\
\hline $\begin{array}{l}\text { Dependiente } \\
\text { rezagada }\end{array}$ & & $0.76^{* * *}$ & & $0.76^{* * *}$ & $0.92^{* * *}$ & $0.92^{* * *}$ & & $-0.29^{* *}$ & & $-0.29^{* *}$ & $-0.30^{* *}$ & $-0.30^{* *}$ \\
\hline \multicolumn{13}{|l|}{$\begin{array}{c}\text { Variables } \\
\text { Neoclásicas }\end{array}$} \\
\hline $\begin{array}{l}\text { CAPITAL per } \\
\text { cápita }\end{array}$ & $0.13^{* *}$ & -0.1 & $0.13^{* *}$ & -0.1 & $-0.38^{* *}$ & $-0.38^{* *}$ & & & & & & \\
\hline $\begin{array}{c}\text { Crecimiento del } \\
\text { CAPITAL per cápita }\end{array}$ & & & & & & & 0.05 & 0.02 & 0.05 & 0.02 & 0.11 & 0.11 \\
\hline \multicolumn{13}{|l|}{$\begin{array}{c}\text { Variables de la } \\
\text { estructura tributaria }\end{array}$} \\
\hline IT/PIB & $6.42^{* * * *}$ & $4.09^{* * *}$ & $6.42^{* * *}$ & $4.09^{* * * *}$ & 2.53 & 2.53 & $-5.20^{* * *}$ & $-5.11^{* * *}$ & $-5.20^{* * *}$ & $-5.11^{* * *}$ & -3.19 & -3.19 \\
\hline $\begin{array}{l}\text { ISR/IT } \\
\text { IVA/IT }\end{array}$ & $-0.41^{* *}$ & $-0.43^{* * *}$ & & & $-0.40^{* *}$ & & $0.34^{* * *}$ & $0.23^{*}$ & & & $0.37^{* *}$ & \\
\hline $\begin{array}{l}\text { IVA/IT } \\
\text { IEPS/IT }\end{array}$ & $-0,5^{* * *}$ & $023^{* * *}$ & $\frac{0.52^{* * *}}{0.06}$ & $\begin{array}{l}0.43^{* * * *} \\
0.1\end{array}$ & $00 * *$ & $\begin{array}{l}0.40^{* *} \\
0.12\end{array}$ & 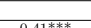 & $035 * * *$ & $-0.34^{* * *}$ & $\begin{array}{l}-0.23^{*} \\
0.0^{*}\end{array}$ & $\Omega^{* * *}$ & $-0.37^{* * *}$ \\
\hline ARANCEL/IT & $\frac{-0.02}{-1.17}$ & $\frac{-0.05}{-0.97^{*}}$ & $\frac{0.00}{-0.64}$ & $\frac{0.1}{-0.53^{*}}$ & $\frac{-0.29}{-0.02}$ & 0.12 & $\frac{0.41}{-0.04}$ & $\frac{0.53}{-0.43}$ & -0.38 & $\frac{0.12}{-0.66}$ & $\frac{0.00}{-1.07^{*}}$ & $\frac{-0.01}{-1.44^{*}}$ \\
\hline \multicolumn{13}{|l|}{$\begin{array}{l}\text { Otras variables } \\
\text { de control }\end{array}$} \\
\hline $\begin{array}{l}\text { Apertura } \\
\text { comercial }\end{array}$ & & & & & 0.21 & 0.21 & & & & & -0.01 & -0.01 \\
\hline $\begin{array}{l}\text { Gasto público } \\
\text { en I+D }\end{array}$ & & & & & -0.02 & -0.02 & & & & & 0.001 & 0.001 \\
\hline Capital humano & & & & & 0.02 & 0.02 & & & & & -0.12 & -0.12 \\
\hline Crisis (dummy) & & & & & 0.02 & 0.02 & & & & & $\begin{array}{ll}-0.01 \\
0.25\end{array}$ & -0.01 \\
\hline Constante & $11.19^{* * *}$ & $4.06^{* * * *}$ & $10.67^{* * *}$ & $3.63^{* * *}$ & $4.86^{* * * *}$ & $4.46^{* * *}$ & -0.07 & -0.0002 & $0.27^{* * *}$ & $0.23^{* * *}$ & -0.35 & 0.02 \\
\hline Se excluye & IVA/IT & IVA/IT & ISR/IT & ISR/IT & IVA/IT & ISR/IT & IVA/IT & IVA/IT & ISR/IT & ISR/IT & IVA/IT & ISR/IT \\
\hline R-cuadrada & 0.4 & 0.66 & 0.4 & 0.66 & 0.73 & 0.73 & 0.39 & 0.45 & 0.39 & 0.45 & 0.59 & 0.59 \\
\hline $\begin{array}{c}\text { R-cuadrada } \\
\text { ajustada }\end{array}$ & 0.33 & 0.61 & 0.33 & 0.61 & 0.62 & 0.62 & 0.3 & 0.36 & 0.3 & 0.36 & 0.43 & 0.43 \\
\hline Durbin-Watson & 0.81 & 2.04 & 0.81 & 2.04 & 1.86 & 1.86 & 2.55 & 2.17 & 2.55 & 2.17 & 2.23 & 2.23 \\
\hline $\begin{array}{c}\text { Breush-Godfrey } \\
\text { (p-value) }\end{array}$ & $21.27(0.00)$ & $0.09(0.76)$ & $21.27(0.00)$ & $0.09(0.76)$ & $0.08(0.77)$ & $0.08(0.77)$ & $3.88(0.05)$ & $0.90(0.34)$ & $3.88(0.05)$ & $0.90(0.34)$ & $1.28(0.26)$ & $1.28(0.26)$ \\
\hline $\begin{array}{c}\text { Jarque-Bera } \\
\text { (p-value) }\end{array}$ & $1.65(0.43)$ & $0.65(0.72)$ & $1.35(0.51)$ & $4.86(0.09)$ & $0.30(0.86)$ & $0.30(0.86)$ & $0.94(0.62)$ & $2.98(0.22)$ & $23.85(6.6 \mathrm{e}-06)$ & $11.64(0.003)$ & $4.35(0.11)$ & $4.35(0.11)$ \\
\hline 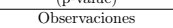 & 45 & 45 & 45 & 45 & 37 & 37 & 44 & 44 & 44 & 44 & 37 & 37 \\
\hline
\end{tabular}

Fuente: Elaboración propia

El impacto del IEPS y de los aranceles sigue siendo incierto. Algunas regresiones sugieren impactos negativos de estos impuestos sobre la productividad, pero en otras regresiones los coeficientes correspondientes pierden significancia estadística, por lo tanto, no hay robustez en los resultados.

En las columnas (7) a (12) de la Tabla 4 se presentan los principales resultados del análisis de regresión cuando la variable dependiente es el crecimiento de la productividad (variación trimestral del PIB/PEA). Los resultados son contradictorios, los coeficientes de ISR/IT tienen signos positivos y estadísticamente significativos, es decir, el ISR favorece la tasa de crecimiento de la productividad. Por el contrario, el IVA/IT tiene impactos negativos y estadísticamente significativos. Lo que implicaría que el ISR impacta negativamente en los niveles de la productividad, pero positivamente en el crecimiento de la productividad, y el IVA tiene los impactos contrarios. Así pues, los impactos sobre el crecimiento del PIB per cápita y el crecimiento del PIB/PEA arrojan resultados contrarios y sin robustez. Solo es posible tener certeza con respecto al impacto de la estructura tributaria sobre los niveles del PIB, por habitante y por habitante en edad de trabajar.

Además de lo ya enunciado y presentado en las Tablas 3 y 4 , se realizaron varias otras pruebas para verificar la robustez de los resultados. Primero, se realizaron ejercicios econométricos cambiando la estructura de los rezagos en las variables independientes. Segundo, se estimaron especificaciones usando las primeras diferencias, tanto de las variables dependientes como de las independientes. Tercero, se analizaron varias otras regresiones en las cuales se incluían o excluían diferentes variables de control. Cuarto, se estimaron las mismas regresiones sin la transformación logarítmica. Quinto, se estimaron las regresiones con los errores estándar robustos de Newey-West. Finalmente, se realizaron estimaciones con la tasa de crecimiento anualizada del PIB per cápita y la productividad, lo que suaviza las fluctuaciones. Después de estas transformaciones los principales hallazgos permanecieron sin cambios sustanciales (estos resultados no se reportan en tablas por cuestiones de espacio). 


\section{Conclusiones}

De acuerdo al análisis de la bibliografía, los impuestos deben tener un impacto negativo, porque reducen el ingreso disponible, pero también son la principal fuente del gasto público. Si lo recaudado en impuestos se gasta correctamente es de esperar un impacto positivo en el nivel de ingreso, aunque cabe no esperarse en países en vías de desarrollo (Marire y Sunde, 2012). No obstante, las pruebas empíricas arrojan resultados inconclusos porque es difícil controlar la doble causalidad y el nexo entre impuestos y gastos públicos (Arnold, 2008; Doménech, 2004). Por otro lado, hay unas pocas pruebas empíricas acerca del impacto de la estructura tributaria sobre el crecimiento económico, es decir, qué tipo de impuestos son favorables para incrementar el nivel de ingreso (PIB), en otras palabras, que impuestos generan menor distorsión a los mercados.

Basados en extensiones del modelo neoclásico de crecimiento económico, los hallazgos previos sugieren que los impuestos al ingreso impactan negativamente sobre el nivel y la tasa de crecimiento del PIB, por el contrario, los impuestos al consumo tienen un impacto positivo (Adkisson y Mohammed, 2014; Arnold, 2008; Arnold et al., 2011; Hakim et al., 2016; Li y Lin, 2015). Por lo tanto, la política fiscal debería privilegiar este tipo de impuesto si el objetivo de política económica se centra en acrecentar el nivel de ingreso.

En esta investigación se realizaron varias pruebas econométricas para evaluar el impacto de la estructura tributaria de México en el periodo 2005 a 2016. Se usaron datos trimestrales y un modelo econométrico neoclásico con extensiones, tal como la literatura empírica lo sugiere. Los hallazgos sugieren que los impuestos sobre el ingreso, el Impuesto Sobre la Renta (ISR) en México, tiene impactos negativos sobre el nivel del PIB per cápita y sobre la productividad (PIB/PEA). Por su parte, el Impuesto al Valor Agregado (IVA) tiene impactos positivos. Es complicado señalar que existe alguna relación causal, pero hay evidencia de que la asociación mencionada existe y concuerda con la mayor parte de los hallazgos previos. No obstante, no se encontró evidencia robusta del impacto de la estructura tributaria sobre la tasa de crecimiento económico, lo que puede interpretarse como evidencia de la presencia de un efecto de largo plazo (sobre niveles), y la ausencia de evidencia para efectos de corto plazo (tasas de crecimiento), lo que coincide con hallazgos previos donde el impacto de largo plazo y de corto plazo puede incluso ser opuesto (Li y Lin, 2015).

En la estructura tributaria de México hay otros dos impuestos relevantes: el Impuesto Especial sobre Producción y Servicios (IEPS) y el impuesto a las importaciones (ARANCEL). Los resultados del análisis de regresión son inconclusos con respecto al impacto que estos impuestos tienen sobre el nivel y la tasa de crecimiento del PIB per cápita y la productividad. No obstante, la contribución a la recaudación fiscal por parte de estos impuestos es baja (alrededor de 10\%) en comparación con el ISR y el IVA (85\%). Por lo tanto, los hallazgos aquí enunciados reflejan el impacto de la parte gruesa de la estructura tributaria mexicana. 
600 REMEF (The Mexican Journal of Economics and Finance)

Impacto de la estructura tributaria sobre el crecimiento económico: El caso de México

\section{Referencias}

Adkisson, R. V., Mohammed, M. (2014). Tax structure and state economic growth during the great recession. Social Science Journal, 51(1), 79-89. http://doi.org/10.1016/j.soscij.2013.10.009

Arnold, J. (2008). Do Tax Structures Affect Aggregate Economic Growth? Empirical Evidence form a Panel of OECD Countries (No. 643). http://doi.org/10.1787/236001777843

Arnold, J., Brys, B., Heady, C., Johansson, Å., Schwellnus, C., Vartia, L. (2011). Tax Policy for Economic Recovery and Growth. The Economic Journal, 121(550), 59-80. http://doi.org/10.1111/j.14680297.2010.02415.x.

Banda-Ortiz, H., Castañeda, A. (2015). Crisis financieras en México (1970-2012) (1ra.). Ciudad de México: Pearson.

Barro, R. J. (1990). Government spending in a simple model of endogenous growth. Journal of Political Economy, 98(5), 103-125.

Chamley, C. (1981). The Welfare Cost of Capital Income Taxation in a Growing Economy. Journal of Political Economy, (89), 468-495.

Doménech, R. (2004). Política Fiscal y Crecimiento Económico. Valencia. Retrieved from https://www . researchgate.net/publication/228694387_Politica_Fiscal_y_Crecimiento_Economico

Doménech, R., García, J. R. (2001). Estructura Fiscal y Crecimiento Económico en la OCDE. Investigaciones Económicas, 25(3), 441-472.

Duncan, D., Peter, K. S. (2012). Unequal inequalities: Do progressive taxes reduce Income Inequality? (Discussion Paper No. 6910).

Edwards, S. (1998). Openness, Productivity and Growth: What Do We Really Know? The Economic Journal, 108(447), 383-398. http://doi.org/10.1111/1468-0297.00293

Gemmell, N. (2001): Fiscal Policy in a Growth Framework. World Institute for development Economics Research Discussion Paper No. 2001/84 Hakim, T. A.,

Karia, A. A., Bujang, I. (2016). Does goods and services tax stimulate economic growth? International evidence. Journal of Business and Retail Management Research, 10(3), 137-147.

Jones, C. I. (1995). R D - Based Models of Econmic Growth.pdf. Journal of Political Economy, 103(4), 759-784.

Jones, L. R., Manuelli, R. E., Rossi, P. (1993). Optimal Taxation in Models of Endogenous Growth. Journal of Political Economy, 3(101), 485-517. Retrieved from http://citeseerx.ist.psu.edu/ viewdoc/download?doi=10.1.1.646.7851\&rep=rep1\&type=pdf

Karras, G. (1999): Taxes and growth: testing the neoclassical and endogenous growth models, Contemporary Economic Policy, ${ }^{\circ}$ 17, pp. 177-188.

Kneller, R., Bleaney, M. F., Gemmell, N. (1999). Fiscal policy and growth: evidence from OECD countries. Journal of Public Economics, (74), 171-190.

Lee, Y., Gordon, R. H. R. H. (2005). Tax structure and economic growth. Journal of Public Economics, 89(5-6), 1027-1043. http://doi.org/10.1016/j.jpubeco.2004.07.002

Li, J. F., Lin, Z. X. (2015). The impact of sales tax on economic growth in the United States: an ARDL bounds testing approach. Applied Economics Letters, 4851(May), 1-5. http://doi.org/10.1080/ 13504851.2015 .1023933

Lucas, R. E. J. (1988). On the mechanics of economic development. Journal of Monetary Economics, $22(1), 3-42$.

Mankiw, N. G., Romer, D., Weil, D. N. (1992). A contribution to the empirics of economic growth. The Quarterly Journal of Economics, 107(2), 407-437. http://doi.org/10.2307/2118477

Mann, A. J. (1980). Economic Development and Tax Structure Change: Mexico, 1895-1975. Public Finance Quarterly, 8(3), 291-306.

Marire, J., Sunde, T. (2012). Economic growth and tax structure in Zimbabwe: 1984-2009. International Journal Economic Policy in Emerging Economies, 5(2), 105-121.

Mendoza, E., Milesi-Ferretti, G., Asea, P. (1997). On The Ineffectiveness of Tax Policy in Altering Longrun Growth: Harberger 's Superneutrality Conjecture. Journal of Public Economic, (66), 99-126.

Myles, G. (2009). Economic growth and the role of taxation - theory (No. 713). Retrieved from http: $/ / \mathrm{dx}$.doi.org/10.1787/2228006336780ECD.

Padovano, F., Galli, E. (2002). Comparing the growth effects of marginal vs average tax rates and progressivity. European Journal of Political Economy, (18), 529-544.

Romer, P. M. (1986). Increasing returns and long-run growth. Journal of Political Economy, (94), 1002-1037.

Romero de Ávila, D. y R. Strauch (2003): Public Finances and Long-term Growth in Europe. Evidence from a Panel Data Analysis. Working Paper No. 246. European Central Bank.

Scully, G. W. (2006) Taxes and Economic Growth. National Center for Policy Analysis. Policy Report No. 292. NCPA 
Revista Mexicana de Economía y Finanzas, Vol. 13 No. 4, (2018), pp. 585-601 601 DOI: http://dx.doi.org/10.21919/remef.v13i4.340

Solow, R. M. (1956). A contribution to the theory of economic growth. Quarterly Journal of Economics, $70(1), 65-94$.

Tomljanocich, M. (2004) The Role of State Fiscal Policy in State Economic Growth Contemporary Economic Policy, 22(3), 318-330.

Tosun, M. S., Abizadeh, S. (2005). Economic growth and tax components: An analysis of tax changes in OECD. Applied Economics, 37(19), 2251-2263. http://doi.org/10.1080/00036840500293813

Tovar, E. D. (2011). Globalización financiera y sus efectos sobre el desarrollo financiero. Ensayos Sobre Política Económica, 29(66), 80-127. Retrieved from http://ideas.repec.org/a/col/000107/010040.html

Tovar-García, E. D. (2012). Financial globalization and financial development in Latin America. Cuadernos de Economía (Colombia), 31(57), 89-112. Retrieved from https://ideas.repec.org/a/col/ 000093/010244.html

Wang, P., Yip, C. . (1992). Taxation and Economic Growth: The Case of Taiwan. American Journal of Economics and Sociology, 51(3), 317-331.

Widmalm, F. (2001). Tax structure and growth: are some taxes better than others? Public Choice, 107(3-4), 199-219. 
602 REMEF (The Mexican Journal of Economics and Finance) 